\title{
Electrochemically deposited poly(3-methylthiophene) performance in single layer photovoltaic devices
}

R. Valaski ${ }^{1}$, L.S. Roman ${ }^{1}$, L. Micaroni ${ }^{2}$, and I.A. Hümmelgen ${ }^{1, a}$

1 Departamento de Física, Universidade Federal do Paraná, Caixa Postal 19044, 81531-990 Curitiba PR, Brazil

2 Departamento de Química, Universidade Federal do Paraná, Caixa Postal 19081, 81531-990 Curitiba PR, Brazil

Eur. Phys. J. E 12, 507 (2003)

Received 26 February 2003

Published online 25 March 2004 - (c) EDP Sciences, Società Italiana di Fisica, Springer-Verlag 2004

Equation (1) on page 508, at the "Results and discussion" section, was mistyped and the correct form is

$$
I P C E=\frac{1.24 \times 10^{-6} J_{s c}}{\lambda I_{\lambda}} .
$$

\footnotetext{
a e-mail: iah@fisica.ufpr.br
} 